\title{
Historia y petite histoire de la $\mathrm{A}(\mathrm{PF}) \mathrm{FUE}$
}

\section{Lafarga Maduell, Francisco}

Universitat de Barcelona, lafarga@ub.edu

\section{Resumen}

Intento de trazar la trayectoria histórica de la actual AFUE, desde los precedentes (a partir de 1975), pasando por su fundación en 1987 y las dificultades de los primeros años de funcionamiento, hasta la refundación en 1992 y el relanzamiento con unas estructuras similares a las actuales. Se trata de las denominaciones, de las personas (los socios, las diversas juntas directivas), los encuentros (en particular los coloquios), así como el interés por la comunicación entre los socios y la visibilidad de la asociación (a través de la página web, de la revista «Çédille» y de otras vías).

Palabras clave: AFUE; Historia.

\section{Résumé}

Cet article est un essai de retracer l'histoire de l'actuelle AFUE, de ses précédents (à partir de 1975), ainsi que de sa fondation en 1987 et les difficultés des premières années de fonctionnement, jusqu'au moment de sa refondation en 1992 et la relance sur des structures nouvelles, qui ont été maintenues pour leur plupart. Le texte fait appel aux diverses dénominations de l'Association, aux personnes (les partenaires, les conseils d'administration), aux rencontres (en particulier les colloques), ainsi qu'à l'intérêt pour la communication entre les partenaires et la visibilité de l'Association (via le site Internet, la revue «Çédille» et autres moyens).

Mots-clés: AFUE ; Histoire.

\begin{abstract}
Attempt to trace the history of the present AFUE, from the preceding (from 1975), through his Foundation in 1987 and the difficulties of the first years of operation, until the Refoundation in 1992 and the relaunch with structures similar to the current. The text refers to appellations, to persons (partners, various boards) and meetings (in particular colloquia), as well as interest in the communication between the partners and the visibility of the Association (via the website, the review «Çédille» and other routes).
\end{abstract}

Keywords: AFUE; History. 
Cuando se cumplen veinticinco años de la refundación de la AFUE conviene echar la vista atrás e intentar trazar una historia de la asociación. Con todo, esa historia empezó mucho antes y estas páginas intentan describirla, necesariamente de modo imperfecto por la propia extensión del artículo. Para ello, lo he dividido en tres partes de desigual extensión, destinadas a recordar la prehistoria, la protohistoria y la historia propiamente dicha, dando a estas expresiones un sentido que no coincide con el fijado por los historiadores, pero que me han servido para denominar tres periodos cronológicos sucesivos que guardan relación entre sí. ${ }^{1}$

\section{La prehistoria (1975-1987)}

La historia del asociacionismo en torno a la situación, promoción y defensa del francés en España empieza en abril de 1975, con la constitución de la Asociación Española de Amigos de la Lengua Francesa. Entre los miembros del núcleo fundador se contaban sonados nombres de la vida cultural y profesional del momento -académicos, profesores, abogados, médicos- como Joaquín Calvo Sotelo, Pedro Laín Entralgo, Luis Lamana de Hoyos, Rafael Lapesa, Fernando Lázaro Carreter, Torcuato Luca de Tena, Julián Marías o Alonso Zamora Vicente.

El año siguiente se creó en el seno de la misma una sección de profesores de francés, que englobaba a numerosos docentes de los distintos niveles de la enseñanza, entre ellos, naturalmente, muchos de universidad.

En 1981, según puede leerse en una descripción inserta en el primer número de la revista Thélème (a la que luego me referiré) la Asociación contaba con no menos de 2.000 asociados, mayoritariamente profesores, aunque también artistas, escritores, funcionarios y profesionales liberales.

En lo que nos toca más de cerca, la Asociación -aparte de la promoción de la lengua y la cultura francesas, acudiendo a instancias como el Ministerio y la Embajada de Francia- colaboró en la edición de Thélème. Revista Española de Estudios Franceses, que fue un intento lamentablemente fallido, por causas económicas y de organización de colaboración además- entre todos los departamentos de Filología Francesa. De hecho, se publicaron únicamente dos números, que sería interesante y justo recuperar del olvido, en 1981 y -el intervalo lo dice todo- 1986, organizados, respectivamente, por la Universidad Complutense y la Universidad de Oviedo.

Otro momento en esta etapa prehistórica fue el encuentro de profesores celebrado entre Valladolid y el castillo de la Mota (Medina del Campo) en febrero de 1985, donde -entre otras cosas- se trató de planes de estudio, la reforma de los cuales ya se había anunciado y produciría un largo debate, que marcó la siguiente etapa.

Y también en este periodo -aunque al margen del asociacionismo- apareció el Repertorio de estudios franceses y provenzales en España, recopilado por Caridad Martínez y yo mismo. Si lo menciono es por su carácter novedoso y porque, andando el tiempo, ese tipo de obra iba a convertirse en uno de los objetivos de la futura APFFUE.

\section{La protohistoria (1987-1992)}

Nutrida en sus primeros momentos por los profesores universitarios de Filología Francesa que se habían inscrito en la Asociación de Amigos de la Lengua Francesa, la APFFUE propiamente dicha se creó en septiembre de 1987 en una reunión celebrada en el Instituto Francés de Madrid, con escasa asistencia, en la que se redactó el acta fundacional, firmada por cinco personas, se aprobó una primera redacción de los estatutos y -sobre todo- se discutió largo y tendido acerca de la reforma de la enseñanza universitaria y de los nuevos planes de estudio de las licenciaturas promovidas por las comisiones de expertos del Ministerio (en nuestro caso, el llamado Grupo XIII). Este tema, que durante meses y meses fue objeto de intenso debate en las universidades, ocupó la mayor parte del tiempo de las reuniones de la incipiente asociación, marcando de este modo -a mi entender- el carácter corporativo de la misma, que con el tiempo fue incorporando aspectos vinculados con la docencia y la investigación.

${ }^{1}$ Debo agradecer la ayuda prestada, con documentos que me han permitido aportar datos concretos y refrescar la memoria, por Manuel Bruña, Julián Muela y José M. Oliver. 
Establecida, pues, el acta fundacional, se procedió a la solicitud de inscripción de la Asociación en el Ministerio del Interior, que no se resolvió favorablemente hasta abril de 1988. Se encargó de todos los trámites Alain Verjat, que era el primer firmante de dicha acta y el convocante de la mencionada reunión fundacional.

Dos meses después de la primera reunión se celebró la segunda, con mayor números de asistentes: en ella se procedió a la elección de la primera Junta Directiva, integrada por Alain Verjat como presidente, Elena Real y Alicia Yllera como vocales, José Ignacio Velázquez como tesorero y Francisco Lafarga como secretario. También se volvió a tratar de las posibilidades de intervención en las decisiones que pudieran tomarse en el Ministerio respecto de los nuevos planes de estudio, en cuanto a criterios de troncalidad, obligatoriedad y opcionalidad, sin olvidar denominaciones, contenidos y porcentajes; y con propuestas y contrapropuestas respecto de posibles licenciaturas distintas de las anunciadas por el Ministerio. Poco se consiguió si se tienen en cuenta la lista definitiva de títulos y las directrices generales de los mismos, que se establecieron en 1990.

Las propias dificultades de la comunicación (todo por correo postal), los retrasos y pérdidas en el abono de las cuotas del casi centenar de miembros, el desánimo causado por el escaso eco ministerial a las propuestas y peticiones emanadas de la Asociación, todo ello hizo que la actividad de la APFFUE languideciera en los últimos meses de su primera etapa. Se imponía una renovación, que se puso de manifiesto en la última asamblea de la misma, en octubre de 1991, uno de cuyos acuerdos - decisivo- fue el firme propósito de revitalizarla con nueva dirección, nuevas ideas y nuevo empuje. Y para ello se emplazó a los asociados a una asamblea y coloquio en la UNED, en abril del siguiente año.

\section{La historia (1992-)}

Para articular la historia de estos últimos veinticinco años me ha parecido más útil y ameno prescindir de la mera sucesión cronológica y avanzar de un modo temático, planteando -espero que con cierto orden- las diversas cuestiones o aspectos que han caracterizado a la asociación, los proyectos y realizaciones más relevantes, los cambios más apreciables decididos por voluntad propia o impuestos por la fuerza de los acontecimientos.

\section{Denominaciones}

Creo que pocas asociaciones han cambiado tanto de nombre en un lapso de tiempo relativamente corto. Empezamos llamándonos APFFUE (Asociación de Profesores de Filología Francesa de la Universidad Española), nombre que ya tenía desde 1987, y que respondía a la denominación de un área de conocimiento (creada en 1984) y a un criterio gremial.

La refundación o relanzamiento de 1992 no comportó cambios en la denominación: había, sin duda, problemas más urgentes y a nadie le pareció prioritario plantearlo. De hecho, fue la segunda junta, elegida en 2001, la que propuso -y tengo que decir en su descargo que por insistencia mía- la modificación de la denominación como Asociación Española de Estudios Franceses, y no sólo porque el acrónimo resultante era más fácil de pronunciar. ${ }^{2}$ En un agradable marco (un precioso hotel en Villajoyosa y un delicioso atardecer de mayo de 2003) y tras una larga hora de discusión, la propuesta se desestimó por tres votos de diferencia frente a una solución de compromiso, la APFUE (o sea, Asociación de Profesores de Francés de la Universidad Española), fórmula que, en realidad, venía a sancionar una doble realidad: la constatación que algunos miembros de la asociación no pertenecían al área de conocimiento con el código 335, y que más de uno que sí estaba en el área no practicaba totalmente, o de una manera ortodoxa, lo que tradicionalmente se ha denominado «Filología Francesa».

Hubo que esperar otros once años (pues once son los que median entre 1992 y 2003) para que se volviera sobre el mismo asunto, aunque desde un punto de partida distinto. Sucedió en Alcalá en 2014, tras un largo periodo de gestación, y en torno a la amenaza -al final desmentida- de la desaparición de la voz «francesista» del diccionario de la RAE. Una larga exposición previa, vía Gaula, y un debate intenso -aunque no tan largo como el de Alicante- dieron como resultado la modificación del nombre en AFUE (Asociación de Francesistas de la Universidad Española). Se regresaba, aunque por otro camino, al fondo o al espíritu de la propuesta desechada en 2003: tal vez habían cambiado los tiempos y las personas,

\footnotetext{
${ }^{2}$ La propia denominación «Estudios Franceses» pareció no gustar a algunos de los asistentes. Los tiempos han cambiado: véase, si no, la denominación adoptada por algunos centros o departamentos para los grados procedentes de licenciaturas en Filología Francesa.
} 
tal vez fuera mayor el poder de convicción del defensor de la propuesta, o tal vez se dieron ambas cosas a la vez. En cualquier caso, la necesaria modificación del artículo 5.2 de los estatutos aclaraba -sin perder de vista dos de las tres acepciones ${ }^{3}$ del vocablo- qué se entendía por «francesista». ${ }^{4}$ Con los estatutos en la mano queda abierta la aceptación de historiadores, geógrafos o sociólogos que estén interesados en el estudio de las realidades de sus materias en los países francófonos; al mismo tiempo, no es condición sine qua non ser profesor o investigador vinculado a una universidad.

\section{Personas}

Aunque primero fuimos «profesores de Filología Francesa», luego «profesores de Francés» y ahora somos «Francesistas», los que formamos esta Asociación somos tanto su razón de ser como su finalidad.

La Asociación empezó con pocos efectivos humanos (126 socios), pero el propio aumento -a pesar de todo- del profesorado, las campañas de captación que se han hecho en algunas ocasiones y el convencimiento de las ventajas de pertenecer a ella han hecho que el número de asociados haya experimentado un espectacular crecimiento, más notable en los primeros años: así, se pasó de los 126 de 1992 a los 268 de 1995, o sea, un aumento del 100\%. El aumento continuó en los años siguientes, hasta alcanzar el máximo en 2009 (495 asociados), y a partir de aquel momento se inició un lento declive hasta llegar a los 460, que es la cifra de mediados de 2016. Este descenso se ha acentuado por las bajas, resultado algunas por aplicación de los estatutos en el caso de impago de cuota, y otras por el aumento del número de jubilaciones o de cambio de orientación profesional.

Precisamente el incremento de las jubilaciones, tanto forzosas como voluntarias, llevó a la Junta a tener que renunciar a la costumbre de proponer el nombramiento de socios de honor a cuantos compañeros se jubilaran, sujetándose desde hace varios años a lo que disponen los estatutos al respecto.

También conviene recordar a los colegas que nos dejaron, algunos ya jubilados, otros en plena actividad docente e investigadora, privando a la Asociación de su presencia, de su espíritu de colaboración y de su afán por contribuir a la consolidación y progreso de los estudios franceses.

Se ha producido asimismo, y eso es muy buena noticia, la incorporación de numerosos profesores e investigadores jóvenes. Precisamente para fomentar la adhesión y participación de los más jóvenes en las actividades de la Asociación se decidió en 2011 y 2013, respectivamente, establecer ayudas para realización de tesis doctorales y para asistencia a los coloquios propios. Con todo, si bien después de la última modificación del nombre puede decirse que somos todos los que estamos, nos hallamos lejos de estar todos los que somos.

Si, como he indicado más arriba, el potencial humano es necesario, no lo es menos la existencia de órganos de gestión. Las sucesivas juntas directivas que han conducido la Asociación durante estos años han llevado a cabo su labor con entusiasmo, consolidando proyectos anteriores, proponiendo nuevas actividades, en definitiva, iniciativas para adecuar la Asociación a las distintas etapas y aprovechar los recursos disponibles, tanto humanos, como económicos y técnicos.

La primera Junta, elegida en 1992 en la asamblea de la UNED, fue reelegida en 1995 (Las Palmas) y 1998 (Cádiz). Estaba compuesta por Alicia Yllera (presidenta), Francisco Lafarga (vicepresidente), Ana González y Arturo Delgado (vocales) y Julián Muela (secretario-tesorero); dos de sus miembros habían pertenecido ya a la junta anterior, elegida en 1987. La segunda Junta se eligió en 2001 (La Laguna) y fue reelegida también en dos ocasiones: 2004 (Oviedo) y 2007 (Lleida): de la primera solo yo decidí continuar, y para ello tuve que organizar una candidatura, en la que aceptaron integrarse Ángeles Sirvent (vicepresidenta), Manuel Bruña y Doina Popa-Liseanu (vocales) y José M. Oliver (secretario-tesorero). Tres mandatos como presidente (más todo lo que llevaba de antes) y la feliz coincidencia con el inicio de mi jubilación anticipada (y de la condición de profesor emérito) me hicieron desistir de pensar siquiera en un nuevo mandato, por más

\footnotetext{
${ }^{3}$ «1. adj. Que tiene influencia francesa. Poesía francesista. Apl. a pers., u. t. c. s. || 2. adj. Que simpatiza con lo francés o lo admira. Apl. a pers., u. t. c. s. || 3. m. y f. Especialista en la lengua y la cultura francesas».

${ }^{4}$ «Art. 5.2. Son socios ordinarios y de pleno derecho los profesores universitarios e investigadores de centros de investigación o de estudios que, dedicándose al estudio de materias que sean propias de los fines de la Asociación, soliciten serlo. Asimismo, también serán socios ordinarios o de pleno derecho los licenciados y estudiantes universitarios de tercer ciclo que, estando seriamente interesados en el estudio de alguno de los temas específicos para los que se funda la Asociación, soliciten ser admitidos como socios, para lo cual deberán ser avalados por dos socios ordinarios con más de tres años de antigüedad». Dichas «materias propias de los fines de la Asociación», aparecen en el artículo 2 de los mismos estatutos: «Art. 2. El objeto específico de la Asociación es estimular, defender e impulsar en España el estudio e investigación de la lengua, la literatura, la historia, la geografía, las instituciones y, en general, la cultura de los países de habla francesa».
} 
que los estatutos no lo impidieran. Por ello, en la asamblea de la Complutense (2010) se presentó una nueva candidatura encabezada por Manuel Bruña, que contaba con J. Oliver como vicepresidente e incorporaba caras «nuevas»: Loreto Cantón y Brigitte Leguen como vocales y Tomás Gonzalo como secretario-tesorero. Cabe decir, para completar esta descripción, que nunca hubo competencia entre candidaturas, lo cual no significa que siempre se diera la unanimidad en el voto: de hecho, solo en una ocasión todos los votos fueron favorables. ${ }^{5}$

Son también los socios los que básicamente sustentan, con sus cuotas, las actividades de la Asociación. Por ello, se ha podido pasar de los 492,41 € (mejor dicho, las 81.902 pta.) con que se arrancó en 1992, a los 42.843,37 € que arroja el balance económico del año 2016. Y eso a pesar de los gastos ocasionados por las ayudas a congresos y publicaciones, o las destinadas a los socios jóvenes, o los producidos por la ampliación y mantenimiento de los recursos electrónicos.

\section{Encuentros}

La sociabilidad, como es sabido, es una de las características del género humano, que comparte con otros animales. En el caso de los socios de la A(PF)FUE tal tendencia se manifiesta en particular en las asambleas y los coloquios.

Ya para el acto refundacional de 1992 se pensó, con acierto, hacer coincidir asamblea y coloquio, pensando que son actividades que se complementan y que cooperan para convocar a los socios.

La historia pormenorizada de las asambleas ocuparía más espacio del razonable en esta historia general de la Asociación. La lectura de las actas resulta muy ilustrativa no solo de las realizaciones y propuestas de la Junta Directiva, o de la situación económica, sino también de las preocupaciones de los socios, de sus propias propuestas; permiten asimismo entrever-¿ipor qué no decirlo?- disparidad de criterios y tensiones.

Los encuentros han propiciado también la movilidad. Ya desde el inicio se pensó en la fórmula de la coorganización entre la propia Asociación y los centros o departamentos, con distinto grado de participación económica -nula en los primeros años por la penuria en que se hallaban las arcas comunes- y de intervención en la toma de decisiones. Por ello los encuentros fueron itinerantes y, además, en algunos casos con varios lugares dentro de la misma sede. Así, en 2003 las asambleas (ordinaria y extraordinaria) se celebraron en el comedor de un hotel de Villajoyosa; el mismo marco, poco académico pero muy sociable, fue el de la asamblea de Salamanca, en un restaurante de La Alberca, y de Almería, en otro de Aguadulce. Otros cambios de lugar fueron los que se produjeron en el encuentro de La Laguna, con la sesión de clausura en el Liceo Taoro de La Orotava; o en el de Oviedo, parte del cual se desarrolló en la antigua Universidad Laboral de Gijón; o en el de Castellón, donde la asamblea se hizo en el salón gótico del castillo de Peñíscola. Lo mismo ocurrió en Zaragoza, pues la asamblea tuvo lugar en la Facultad de Ciencias Humanas y de la Educación de Huesca, o en Jaén, donde algunos actos y la asamblea se celebraron en el palacio de Jabalquinto de Baeza, o en Almería, con el traslado de los bártulos al castillo de Roquetas de Mar. En el caso de Valladolid no salimos de la ciudad, aunque lo parecía, ya que nos trasladamos en barco al Museo de la Ciencia, donde se celebró la última sesión. En rigor, el único coloquio organizado conjuntamente por dos universidades fue el de 2012, con actos repartidos entre la Universitat de Barcelona y la Universitat Autònoma de Barcelona.

Por otra parte, los coloquios han sido en ocasiones el marco para rendir homenaje a compañeros con motivo de su jubilación. Así ocurrió en el de 2004 en Oviedo con Ma Aurora Aragón, en el de 2005 en Valladolid con Francisco J. Hernández, en 2010 en la Complutense con Javier del Prado y en 2012 en Barcelona conmigo mismo. Además, como el coloquio de Zaragoza coincidió con el acto de investidura de doctor honoris causa de Alicia Yllera, resultó también una celebración con muchos de nosotros presentes en un día tan trascendente para ella.

\footnotetext{
${ }^{5}$ Tengo que añadir que la primera Junta y más, si cabe, la segunda fueron reuniones de amigos. De la primera recuerdo los viajes a Madrid, donde nos reuníamos normalmente en dos ocasiones entre asamblea y asamblea: como yo solía viajar en el tren nocturno, llegaba muy temprano a Chamartín, con lo cual podía ir tranquilamente en metro hasta Moncloa y de allí bajar caminando por el parque del Oeste hasta la UNED en Senda del Rey. Las reuniones eran muy amenas, de trabajo pero también de conversación amistosa, favorecida por los largos ratos que debíamos dedicar a doblar convocatorias, a ensobrar boletines y a pegar etiquetas con direcciones. Nos daba la hora de la comida, que muchas veces, por insistencia del tesorero (por ahorrar, más que nada), hacíamos en la cafetería de la Facultad: eso sí, en un comedor reservado para profesores donde ponían un menú aceptable y a precio módico. Con la segunda Junta el modus operandi cambió: como nos habíamos modernizado todos (incluso yo) podíamos comunicarnos por correo electrónico, funcionaba el sistema de los corresponsales en los centros, el proceso de envíos se había simplificado: en fin, que no fueron necesarias tantas reuniones presenciales. Con todo, siempre había una entre asambleas, y nos propusimos ir cambiando de sede, por aquello de huir del centralismo madrileño, además de favorecer el turismo interior. También nos permitía hacer reuniones más prolongadas y distendidas, aprovechando distintos momentos del día. En cualquier caso, a pesar del trabajo y la responsabilidad, recuerdo esos años con mucho cariño.
} 
Los coloquios, ¡qué duda cabe!, han sido uno de los motores de la Asociación. A la itinerancia ya señalada hay que agregar otras consideraciones y algunas cuestiones que fueron planteándose a lo largo de los años.

En primer lugar conviene señalar que el número de participantes ha sido siempre razonablemente elevado, con oscilaciones debidas a hechos coyunturales. Eso no impidió que en varias ocasiones (y la primera ya en 1994) se planteara por algunos socios la conveniencia de celebrar coloquios bianuales; pero el hecho de que igualmente debía convocarse la asamblea aconsejó seguir con la frecuencia anual, por lo menos mientras no descendiera de modo alarmante el número de participantes... y se han celebrado ya veinticinco. También se planteó en varias ocasiones si el coloquio debía reservarse a los socios o abrirse a participantes externos; no llegó a tomarse decisión alguna al respecto, dejándose la decisión al criterio de los organizadores de cada coloquio. De hecho, el primero en el que participaron comunicantes ajenos a la Asociación fue el de La Laguna en 2001, situación que se ha repetido en los coloquios siguientes. También se ha dejado siempre al criterio de los organizadores la adopción de la temática del coloquio, aunque con el ruego de que fuera amplia, de modo que no impidiera la participación de todos los socios que lo desearan.

La implicación de la Asociación en los coloquios se ha traducido asimismo en las aportaciones económicas tanto a la organización de los mismos como a su publicación; esas ayudas, que no se aprobaron hasta 1998, tuvieron también como consecuencia que se liberara a los socios del abono de la cuota de inscripción, reservada luego únicamente a los no socios. Cabe decir que en 2010, recogiendo las experiencias de los organizadores y otros temas planteados en las asambleas, la Junta Directiva estableció un protocolo que pudiera servir de guía para futuros encuentros.

Y no podía olvidar el mencionar los complementos preparados por los organizadores: las visitas culturales y las cenas. Algunas son para mí memorables, por lo novedosas o sorpresivas, o por lo espectaculares. Así, los paseos en barco en Valladolid y Huelva; el recorrido por el románico de La Rioja (bajo la lluvia, eso sí); la visita del Jardín Botánico de Gijón; las salidas ya mencionadas - mitad trabajo mitad asueto- en Almería, Jaén, Castellón... y algunas otras excursiones que me dejo en el tintero. Y qué decir de los ágapes, más recordados por la compañía que por la gastronomía, pero recordados al fin. Por las sorpresas que depararon me vienen a la memoria la cena de La Laguna, con el nombramiento de las «mises» y los «místeres» del coloquio; o la de Logroño, que aquello parecía una boda, con baile incluido; o la de Alcalá, con la grata sorpresa de la cantante que nos hizo tararear canciones de nuestros recuerdos; o la de Almería, con la demostración de danza por parte de profesionales y amateurs.

Además de los coloquios anuales propios, la Asociación ha colaborado en la organización y/o en la publicación de resultados de los coloquios internacionales de Lingüística Francesa, que van ya por la undécima edición, así como en coloquios celebrados con otras asociaciones, de los que se han llevado a cabo seis hasta el momento.

\section{Comunicación}

La comunicación, esencial para el buen funcionamiento de cualquier empresa en común, fue preocupación de la Asociación y de su Junta Directiva desde los inicios. Una de las primeras herramientas que se activaron fue el Boletín, que yo mismo había propuesto en la etapa protohistórica y que se planteó en serio y se decidió en la asamblea de 1993. De hecho, el primer número apareció en mayo de ese año y fue saliendo anualmente hasta 1997 (con el n 6). Constaba de secciones fijas (acta de la última asamblea, movimiento de socios, reseñas y anuncios de coloquios y actividades, relación de tesis defendidas, mención de revistas y grupos de trabajo, resumen de publicaciones recientes). Las dificultades de su elaboración, pues en gran parte dependía de las informaciones facilitadas por los socios, el coste económico que suponía y lo engorroso de su envío, decidieron a la Junta a suprimirlo, con la esperanza de que pronto podrían incluirse sus contenidos en una proyectada página web que, de hecho, no fue realidad hasta 2002.

También para facilitar la comunicación entre los socios y la Junta Directiva se creó en 1995 la figura del corresponsal (o, como se denominó al principio, coordinador de información) con el encargo de comunicar a la Junta las actividades de su centro o departamento vinculadas con los estudios franceses. Con el tiempo se les pidió también a los corresponsales que se ocuparan de transmitir a sus compañeros las informaciones o los envíos postales procedentes de la Asociación.

Y, tercer elemento en la línea comunicativa, en 2004 se creó la lista de difusión Gaula, integrada en RedIRIS, que ha servido para garantizar una comunicación ágil y rápida desde la Junta Directiva hacia los socios y de los socios entre sí, sirviendo de escaparate para anunciar congresos, actividades y publicaciones, y de foro donde compartir opiniones y 
mantener debates. Como sabemos, ha habido en ocasiones debates que han degenerado en un largo rosario de dimes y diretes, no siempre de interés general; mientras que en otras la impericia o el descuido han hecho que se colaran comentarios o noticias de índole personal, cuando no íntima.

\section{Visibilidad}

La visibilidad ha sido uno de los grandes retos a los que se ha enfrentado la Asociación y -a tenor de los resultadosparece que se ha cumplido.

El primer frente fue el de recopilar y hacer pública la labor investigadora en torno al francés. En realidad, la primerísima versión de un catálogo o repertorio bibliográfico es anterior a la propia creación de la Asociación e 1987: se trata del Repertorio de estudios franceses y provenzales en España, que reuní con mi compañera y amiga Caridad Martínez. El propio título refleja, a mi entender, el espíritu abierto de la empresa, su independencia respecto de cualquier área de conocimiento y de cualquier grupo profesional. Por tal motivo se hallan también en sus páginas trabajos de investigadores no vinculados a la Filología Francesa o a los Estudios Franceses. Conviene añadir que fue una obra hecha a mano, con la información circulando por correo postal.

Parece que los esfuerzos no fueron en balde y que el repertorio -con todas sus carencias o, mejor, sus ausencias- resultó útil. De hecho, la idea de continuarlo y ampliarlo fue unas las primeras decisiones tomadas por la Junta de 1992; así, en 1994 apareció un volumen que recogía los contenidos del de 1987, con adiciones hasta 1993 y con una nueva presentación e índices, preparado por Alicia Yllera y Julián Muela, en el que aparecían también los nombres de los recopiladores del primero. El título, para adecuarlo a la realidad de la Asociación en el seno de la cual se publicaba, se cambió por el de Repertorio de estudios franceses en la Universidad española, que es el mismo que presentó en 2003 una nueva edición, esta vez a cargo únicamente de A. Yllera y J. Muela, que recogía lo comunicado por los autores para el periodo 19932000 .

Obviamente, una recopilación de esas características con un complejo sistema de claves temáticas, tenía más su lugar según avanzaban los tiempos- en una base de datos en línea que en un volumen en papel. Por eso se decidió integrar todo lo recopilado en la página web que ya se había creado, actualizando de paso los datos. El proceso de trasvase resultó más complejo de lo previsto y la comunicación de los datos por parte de los socios más lenta de lo razonable: de hecho, no pudo ponerse totalmente en acceso abierto hasta 2008, esta vez con la denominación de Catálogo Hispánico de Estudios Franceses, que tenía la ventaja de dar un acrónimo pronunciable y, además, con significado. Pero, una vez más, una buena idea se ha visto empañada por la relativa colaboración de quienes debían estar más interesados en que el catálogo fuera completo, pues de lo contrario no cumple su cometido.

Algo parecido ha sucedido con el directorio de socios. Durante unos años, en el periodo de existencia del Boletín, se incluyeron en él los datos personales mínimos de los socios que iban incorporándose. Una vez cerrado el Boletín (1997), se pensó en publicar un directorio general en formato libro. Hubo para ello que solicitar nuevos datos a los socios (tanto a los nuevos como a los antiguos), pues el formato de la ficha se había modificado. El trabajo de recuperación de datos y de edición del directorio, que corrió a cargo de Doina Popa-Liseanu, se fue alargando en el tiempo más de lo debido por las causas apuntadas y no apareció hasta mayo de 2003.

El largo periodo de gestación hizo que su aparición siguiera a la apertura de la página web, en la que debía integrarse naturalmente la información que contenía. La página web ha sido la realización estrella, no solo por su complejidad, sino porque asegura la mayor visibilidad a la Asociación y a todo lo que hace y representa. También su prehistoria fue larga, pues ya en 1996 se planteó en asamblea la necesidad de establecerla, aunque hasta 2002 no estuvo operativa, gracias al esfuerzo de José M. Oliver. Se incluyeron en ella paulatinamente las herramientas de información y difusión ya aparecidas en otros formatos, como el repertorio de estudios, el directorio, así como información sobre actividades de la propia Asociación y de sus miembros. La página, por supuesto, ha ido evolucionando, ganando en imagen, presentación, contenidos y prestaciones.

La creación de una revista propia de la Asociación no fue, al contrario, un asunto considerado urgente durante sus primeros años de vida. Tal vez el fracaso de la primera Thélème aconsejaba meditar muy bien la posibilidad de creación de una revista propia, habida cuenta -por otra parte- que en el ínterin habían visto la luz varias revistas de la especialidad. De 
hecho, incluso se llegó a plantear en la asamblea de 1997 aplazar su hipotética puesta en marcha. Hasta 2001 no se propuso en firme estudiar su viabilidad, creándose al efecto un grupo de trabajo, que el año siguiente ya pudo presentar resultados concretos: carácter digital, denominación, contenidos, comité editorial, etc. Finalmente, en 2005 salió el primer número de Çédille. Revista de estudios franceses y, desde entonces, puntualmente en el mes de abril, se abre el acceso a una nueva entrega. Cuando la revista tenía ya cierto recorrido se creó una serie de Monografías, también en formato electrónico, que consta en la actualidad de cinco volúmenes. Gracias a sus propios méritos, pero también -y mucho- al tesón y buen hacer de su director, J. M. Oliver, Çédille goza de numerosos reconocimientos y está incluida en los principales índices y directorios. Su crecimiento, favorecido a buen seguro por su indexación, ha sido espectacular: de los siete artículos del $\mathrm{n}^{\circ}$ 1 (2005) se ha pasado a los 43 del no 12 (2016).

La publicación de los coloquios contribuye asimismo a proyectar y hacer visibles nuestros trabajos, por más que esa vía de publicación sea poco o nada valorada por las agencias y comisiones de evaluación. Un paso notable en la visibilidad de estas obras colectivas ha sido la incorporación de las mismas a un portal de prestigio. Al no llegar a buen puerto la propuesta presentada a la Biblioteca Virtual Miguel de Cervantes, en 2008 se planteó a Dialnet, que aceptó incluir nuestros coloquios siempre que se facilitara una versión digitalizada y se obtuvieran las autorizaciones de los editores o derechohabientes. Una vez cumplidas todas esas formalidades se incorporaron en 2012 los primeros volúmenes, y en los años sucesivos se han agregado los disponibles, una vez cumplidos esos trámites. Lo mismo se ha hecho con los volúmenes de actas de los congresos de Lingüística Francesa.

\section{Profesión}

El sesgo netamente gremial que adoptó la Asociación desde sus inicios ha hecho que la preocupación por la formación y estabilidad laboral del profesorado, así como la situación de los estudios de Filología Francesa sea una cuestión recurrente tanto en las asambleas como en diversas iniciativas que se han adoptado. Al mismo tiempo, ha sido constante la inquietud por el estado del francés en los demás niveles de la enseñanza. En este sentido, cabe destacar las relaciones que se han mantenido con otras asociaciones y entidades, así como la integración de la AFUE en la Federación de Asociaciones de Francés.

En lo que respecta a la enseñanza universitaria, que es nuestro ámbito profesional, el debate sobre la realidad de los estudios está presente desde la propia creación de la Asociación en 1987, ante la inminencia de una nueva reforma de la enseñanza superior, con la creación de nuevas titulaciones. Aun cuando poco se pudo hacer de manera conjunta, debido a las soluciones que cada centro o departamento hubo de adoptar para adaptarse a la nueva situación, una vez aprobados los distintos planes de estudios se llevó a cabo toda una labor de recogida de datos sobre la situación en cada universidad, con lo que finalmente pudo lograrse un mapa bastante fiel de la misma. Y más adelante se reavivó el debate con motivo de la aplicación del EEES y la creación de los grados. En todo este proceso de dinamización y recogida de datos jugaron un papel primordial, por parte de la Junta, Ana González, Ángeles Sirvent y Manuel Bruña. Como muestra de esa preocupación general, en varios encuentros se han previsto sesiones de exposición y debate acerca de la situación de los estudios.

En el ámbito profesional pueden situarse asimismo las gestiones y contactos habidos con varias representaciones de países francófonos y, en particular, con la Embajada de Francia: tras varios años de desencuentros o de falta de comunicación se reanudaron los contactos con los Servicios Culturales de la Embajada y en particular con el attaché de cooperación lingüística de turno. Por lo que mí respecta, guardo muy buen recuerdo del trato con Yannick Rascouët, Fabienne Lallement y, ya en el último año, con Pascal Sanchez. La colaboración de la embajada se tradujo en ayudas -directas o indirectas- a la organización de actividades de la Asociación (y así consta en los resultados de las mismas) y en presencias en coloquios y encuentros.

\section{Colaboraciones}

Una vez consolidada, la Asociación se propuso, a través de su Junta Directiva, crear contactos y principios de colaboración con otras asociaciones. Así, en 2004 se estableció un convenio de colaboración con la SHF-Société des Hispanistes Français de l'Enseignement Supérieur, que era -aunque en negativo, podría decirse- la contraparte de nuestra Asociación 
en Francia. El acuerdo se hizo bajo los auspicios de la Embajada, a partir de una entrevista mía con el embajador, y se vio favorecido, como tantas veces ocurre, por una relación personal, que tenía con el entonces presidente de la SHF, Jacques Soubeyroux. La principal consecuencia del acuerdo fue la convocatoria de coloquios bilaterales, que debían celebrarse trianualmente. El primero de ellos, en efecto, tuvo lugar en Sevilla a finales de 2006 y resultó muy concurrido; menor asistencia, sin embargo, registró el organizado en la ENS de Lyon en 2008, organizado por la contraparte francesa.

Mientras tanto se habían establecido vínculos con la joven APEF-Associação Portuguesa de Estudos Franceses, a partir de un primer encuentro personal con Ana Clara Santos, una de las promotoras, y de las más activas, de su asociación. La voluntad de colaboración se afianzó en Sevilla, durante el coloquio ya mencionado entre la APFUE y la SHF de 2005, y se concretó el año siguiente con la participación de varias colegas portuguesas en una mesa redonda sobre situación de los estudios de francés en ambos países, en el marco del coloquio de Huelva y en el establecimiento de coloquios conjuntos bianuales el primero de los cuales se celebró en Faro en 2007 y el segundo en Barcelona en 2009.

Puesto que la frecuencia adoptada para cada coloquio conjunto hacía que en 2011 coincidieran ambos encuentros, se acordó que fueran a tres bandas en lo sucesivo, y así nacieron los coloquios de Faro de ese año, con una participación que sorprendió a los organizadores, y el de Santiago de 2014.

También en el ámbito de la colaboración debe mencionarse la que se inició en 2005 con la Association Internationale d’Études Québéquoises con el objeto de canalizar las candidaturas españolas a las becas para estancias en Quebec; con todo, parece que se ha interrumpido en el último año, al no haber recibido la solicitud que cursaba dicha asociación.

\section{Final}

La historia de la asociación a partir de 1992 está marcada por un innegable avance en los distintos ámbitos o líneas de actuación previstos, en una mayor visibilidad, gracias - entre otras cosas-a la creación de la página web, a una más fluida comunicación, tanto entre la Junta Directiva y los asociados como entre los propios miembros (a través de Gaula). También ha crecido durante todos estos años el número de asociados y, gracias a sus cuotas, así como a una relativa contención del gasto, la Asociación cuenta con una saneada economía. Con todo, le quedan todavía algunos asuntos pendientes: si en el plano de la investigación los logros en el ámbito de la Filología Francesa -o de los Estudios Francesesson obvios (con numerosas publicaciones, frecuentes coloquios, revistas especializadas y cada vez mejor situadas en las listas de índices de impacto: o sea, una visibilidad cada vez mayor), en el de la docencia está claro que se ha ido perdiendo no solo el lugar que se tuvo en el pasado, sino incluso visibilidad, al no aparecer siquiera en las denominaciones de algunos grados, y no por desidia de sus promotores, sino por exigencias de programación y de expectativas de resultados.

Soy consciente de que el recorrido que he realizado es apresurado e incompleto, y que la Asociación merece mayor atención y mejor trato. La historia, la gran historia queda, pues, por escribir, con más datos, más concreciones, mejores argumentos. A esa tarea puede contribuir el archivo de documentos que se ha reunido y que se custodia en la Universidad de Alicante, junto con programas, carteles e imágenes. 